\title{
Heterogeneity of Auditory Verbal Working Memory in Schizophrenia
}

\author{
Gerard E. Bruder, Daniel M. Alschuler, \\ Christopher J. Kroppmann, Shiva Fekri, \\ Roberto B. Gil, Lars F. Jarskog, \\ Jill M. Harkavy-Friedman, Raymond \\ Goetz, and Jürgen Kayser
}

New York State Psychiatric Institute, New York, New York, and Columbia University College of Physicians and Surgeons

\author{
Bruce E. Wexler \\ Connecticut Mental Health Center, New Haven, \\ Connecticut, and Yale University
}

\begin{abstract}
The heterogeneity of schizophrenia remains an obstacle for understanding its pathophysiology. Studies using a tone discrimination screening test to classify patients have found evidence for 2 subgroups having either a specific deficit in verbal working memory (WM) or deficits in both verbal and nonverbal memory. This study aimed to (a) replicate in larger samples differences between these subgroups in auditory verbal WM; (b) evaluate their performance on tests of explicit memory and sustained attention; (c) determine the relation of verbal WM deficits to auditory hallucinations and other symptoms; and (d) examine medication effects. The verbal WM and tone discrimination performance did not differ between medicated $(n=45)$ and unmedicated $(n=38)$ patients. Patients with schizophrenia who passed the tone screening test (discriminators; $n=60$ ) were compared with those who did not (nondiscriminators; $n=23)$ and healthy controls $(n=47)$. The discriminator subgroup showed poorer verbal WM than did controls and a deficit in verbal but not visual memory on the Wechsler Memory Scale-Revised (Wechsler, 1987), whereas the nondiscriminator subgroup showed overall poorer performance on both verbal and nonverbal tests and a marked deficit in sustained attention. Verbal WM deficits in discriminators were correlated with auditory hallucinations but not with negative symptoms. The results are consistent with a verbal memory deficit in a subgroup of schizophrenia having intact auditory perception, which may stem from dysfunction of language-related cortical regions, and a more generalized cognitive deficit in a subgroup having auditory perceptual and attentional dysfunction.
\end{abstract}

Keywords: schizophrenia, working memory, auditory perception, attention, hallucinations

The clinical and neurocognitive heterogeneity of schizophrenia remains an obstacle to understanding its pathophysiology. Numerous studies have demonstrated deficits in working memory (WM) in patients with schizophrenia using visual (Barch, Csernansky,

This article was published Online First January 10, 2011.

Gerard E. Bruder, Daniel M. Alschuler, Christopher J. Kroppmann, Shiva Fekri, and Jürgen Kayser, Cognitive Neuroscience Division, New York State Psychiatric Institute, New York, New York, and Department of Psychiatry, Columbia University College of Physicians and Surgeons; Roberto B. Gil, Translational Imaging Division, New York State Psychiatric Institute, and Department of Psychiatry, Columbia University College of Physicians and Surgeons; Lars F. Jarskog, Lieber Center Clinical Services Division, New York State Psychiatric Institute, and Department of Psychiatry, Columbia University College of Physicians and Surgeons; Jill M. Harkavy-Friedman and Raymond Goetz, Clinical Phenomenology Division, New York State Psychiatric Institute, and Department of Psychiatry, Columbia University College of Physicians and Surgeons; Bruce E. Wexler, Connecticut Mental Health Center, New Haven, Connecticut, and Department of Psychiatry, Yale University.

This research was supported by Grant MH066597 from the National Institute of Mental Health. We thank John Keilp for his help with the neuropsychological tests.

Correspondence concerning this article should be addressed to Gerard E. Bruder, Cognitive Neuroscience Division, Unit 50, New York State Psychiatric Institute, 1051 Riverside Drive, New York, NY 10032. E-mail: bruderg@pi.cpmc.columbia.edu
Conturo, \& Snyder, 2002; Callicott et al., 2000; Carter et al., 1998; Park \& Holzman, 1992; Perlstein, Carter, Noll, \& Cohen, 2001) and auditory (Gold, Carpenter, Randolph, Goldberg, \& Weinberger, 1997; Menon, Anagnoson, Mathalon, Glover, \& Pfefferbaum, 2001; Wexler, Stevens, Bowers, Sernyak, \& GoldmanRakic, 1998) tasks, but few have addressed the issue of individual differences between patients in the nature of their deficits. It has been suggested that subgroups of patients in the general diagnostic category of schizophrenia are marked by differences in their cognitive deficits and that such "cognitive phenotypes" may be more homogeneous in clinical and pathobiological characteristics (Egan et al., 2001, p. 104).

In a study of auditory WM, Wexler et al. (1998) reasoned that it is important to distinguish between patients who might perform poorly because they could not adequately attend to or perceive the auditory stimuli and those who have intact attention and perception. They divided patients having schizophrenia into two subgroups on the basis of their performance on a tone discrimination test requiring auditory perception and attention. Patients who performed normally on the tone discrimination test (i.e., discriminators [Ds]), showed normal performance on a nonverbal WM test (i.e., tone serial position test) but showed a deficit on a parallel verbal WM test (i.e., Word Serial Position Test [WSPT; Wexler et al., 1998], which involves storage and rehearsal of phonological and sequential information over a delay period). In contrast, patients who performed poorly on the tone discrimination test (i.e., nondiscriminators [NDs]) had marked deficits on both word and 
tone WM tests. Wexler et al. suggested that the global performance deficit in this group may stem from a perceptual or encoding dysfunction early in the auditory processing sequence. Bruder, Wexler, Sage, Gil, and Gorman (2004) confirmed the difference in WSPT performance between the D and ND subgroups and found that the verbal memory deficit in D patients extended to learning and recall of verbal material on the Wechsler Memory ScaleRevised (WMS-R; Wechsler, 1987). In contrast to D patients, who showed poorer verbal than visual memory scores on the WMS-R, ND patients showed poor performance on both verbal and visual indices. Although the D and ND subgroups did not differ in severity of positive symptoms, ND patients had greater negative symptoms than did D patients on the Positive and Negative Symptom Scale (PANSS; Kay, Opler, \& Fishbein, 1999).

Impairments shown on neuropsychological tests of cognitive function generally have only small to moderate correlations with severity of negative symptoms (Harvey, Koren, Reichenberg, \& Bowie, 2006). Deficits in visuospatial WM have been consistently found to be related to negative symptoms of schizophrenia (Carter, Robertson, Nordahl, Chaderjian, \& Oshora-Celaya, 1996; Gooding \& Tallent, 2002; Park, Puschel, Sauter, Rentsch, \& Hell, 1999). There is, however, less agreement on the relationship between auditory verbal WM and symptom features. Thus, performance of patients with schizophrenia on the Letter-Number Sequencing Test (Tulsky, Zhu, \& Ledbetter, 1997; Wechsler, 1997) was negatively correlated with scores on the Scale for the Assessment of Negative Symptoms (SANS; Andreasen, 1983) after controlling for the influence of the Wechsler Adult Intelligence Scale-Revised (Wechsler, 1981) vocabulary or sustained attention (Perry et al., 2001), but in another study, letter-number performance was not associated with negative symptoms on the PANSS (Donohoe, Corvin, \& Robertson, 2006). Stevens, Donegan, Anderson, Goldman-Rakic, and Wexler (2000) found that, among patients with schizophrenia who performed normally on the tone discrimination test (i.e., D patients), poorer performance on the WSPT was significantly associated with severity of positive but not negative symptoms on the PANSS. Neuroimaging studies also indicate that severity of positive symptoms of schizophrenia, in particular auditory hallucinations or delusions, is associated with activation of language-related regions during verbal WM tasks (Hashimoto, Lee, Preus, McCarley, \& Wible, 2010; Wible et al., 2009). These conflicting findings concerning the relationship between verbal WM deficits and symptoms of schizophrenia could arise from a failure to take general cognitive impairment of patients into account (Donohoe et al., 2006) or from a problem with the symptom measures, which may be particularly true for negative symptoms.

The present study aimed to replicate in larger samples the difference in auditory verbal WM between D and ND subgroups on the WSPT (Bruder et al., 2004; Wexler et al., 1998) and to examine medication effects by comparing the tone discrimination and WSPT performance of patients on antipsychotics versus off antipsychotics. To further evaluate the material specificity of memory deficits in D and ND subgroups, we again compared their performance on verbal and nonverbal indices on the WMS-R. Moreover, patients were tested on the Letter-Number Sequencing Test (Tulsky, Zhu, \& Ledbetter, 1997; Wechsler, 1997) and the Continuous Performance Test-Identical Pairs (CPT-IP; Cornblatt \& Keilp, 1994), so as to further assess the difference between D and ND subgroups in verbal WM and sustained attention. We also aimed to replicate our finding of greater negative symptoms in ND than D patients and to examine the relation of verbal WM deficits on the WSPT to positive and negative symptoms of patients in these subgroups. On the basis of the findings of Wible et al. (2009), suggesting that auditory hallucinations may interfere with verbal WM processing, we hypothesized that D patients having auditory hallucinations would show greater deficits on the WSPT when compared with those without hallucinations.

\section{Method}

\section{Participants}

Seventy-four inpatients from the psychosis research unit and 30 outpatients from the Lieber Center Recovery Clinic at the New York State Psychiatric Institute were recruited for the study. Four patients were excluded because of comorbid medical, neurological, or substance abuse problems, and six patients were excluded because they had a hearing loss or did not complete the tone discrimination test (see later). An additional 11 patients were excluded because they did not meet the criteria for schizophrenia or schizoaffective disorder. The remaining 83 patients (49 male, 34 female) met criteria for schizophrenia $(n=60)$ or schizoaffective disorder (bipolar type, $n=14$; depressive type, $n=9$ ) according to the Diagnostic and Statistical Manual of Mental Disorders (4th ed.; DSM-IV; American Psychiatric Association, 2000). ${ }^{1}$ Most patients $(n=70)$ received a semistructured interview by a trained and reliable rater using the Diagnostic Interview for Genetic Studies (DIGS; Nurnberger et al., 1994), which was developed in the National Institute of Mental Health Human Genetics Initiative collaboration. It combines items from commonly used research instruments, including clinical rating scales (e.g., SANS [Andreasen, 1983] and the Scale for the Assessment of Positive Symptoms [SAPS; Andreasen, 1984]), the Schedule for Affective Disorders and Schizophrenia (Endicott \& Spitzer, 1978), and the Structured Clinical Interview for DSM-III-R and IV (SCID; Spitzer, Williams, Gibbon, \& First, 1990; and First, Spitzer, Gibbon, \& Williams, 2002, respectively). The DIGS has undergone extensive reliability testing with good results. DSM-IV research

\footnotetext{
${ }^{1}$ Patients meeting criteria for either schizophrenia or schizoaffective disorder were included because we did not find a significant difference in their tone discrimination or WSPT performance. Nor did they differ in gender, age, education, or handedness. The percentage of correct responses on the tone discrimination test was analyzed using a Group (schizophrenia, schizoaffective, controls) $\times$ Gender (male, female) $\times$ Ratio of Tones (.67, $.75, .85, .90, .95,1.00)$ repeated measures analysis of variance (ANOVA) There was a significant difference in tone discrimination among groups, $\mathrm{F}$ $(2,124)=8.40, p<.001$. Both patients with schizophrenia $(M=82.8$, $S D=18.6)$ and schizoaffective disorder $(M=84.6, S D=18.2)$ showed poorer tone discrimination than controls $(M=95.1, S D=9.3$, both $p<$ $.05)$, but there was no significant difference between the schizophrenia and schizoaffective groups. The same was true for the WSPT. There was a significant difference in WSPT accuracy among groups, F $(2,124)=$ $22.58, p<.001$, with both patients with schizophrenia $(M=75.4, S D=$ $19.0)$ and schizoaffective $(M=81.0, S D=11.0)$ performing more poorly than controls $(M=94.1, S D=6.8$, both $p<.05)$, but there was no significant difference between the schizophrenia and schizoaffective group.
} 
diagnoses for the 70 patients interviewed with the DIGS were made by a consensus of at least two doctoral-level research clinicians $(\mathrm{MD}$ or $\mathrm{PhD}$ ) and the clinical research interviewer during regular consensus conferences. The DSM-IV diagnoses of the remaining 13 patients were made by psychiatrists on the research units. ${ }^{2}$ Symptom ratings were also obtained using the PANSS (Kay et al., 1999). Master's-level raters were required to achieve adequately high interrater reliability with each other (interclass correlations of greater than .85 for symptom ratings). A total Brief Psychiatric Rating Scale (BPRS; Overall, 1974) score was derived from the 18 PANSS items that match those in the BPRS. When tested, 45 patients were receiving risperidone $(n=12)$, aripriprazole $(n=11)$, ziprasidone $(n=11)$, olanzapine $(n=4)$, quetiapine $(n=4)$, or clozapine $(n=3)$. The remaining 38 patients did not receive antipsychotic medications for about 2 or more weeks before testing.

A control group consisted of 52 healthy adults (23 male) who were recruited from the New York metropolitan area and paid \$15 per hour for participation. Outpatients were also paid $\$ 15$ per hour, whereas inpatients received treatment on the research unit but were not paid for their participation. ${ }^{3}$ Controls were interviewed using the Structured Clinical Interview for DSM-IV Axis I Disorders (nonpatient edition; First, Spitzer, Gibbon, \& Williams, 1996) to exclude those with current or past psychopathology. Both patients and controls were excluded if they had a history of neurologic insult or illness. Patients were excluded for current substance abuse or past substance dependence sufficient to obscure the diagnosis of schizophrenia, and controls were excluded for past or current substance abuse or dependence. All participants completed audiograms, and they were excluded if the average hearing loss at 500, 1000, and $2000 \mathrm{~Hz}$ was greater than $30 \mathrm{~dB}$ in either ear or differed by $10 \mathrm{~dB}$ or more between ears. After being given a description of the study, participants provided written informed consent before we initiated testing following procedures approved by the Columbia University Institutional Review Board.

\section{Measures}

Tone discrimination screening test. The tone discrimination test (Wexler, Donegan, Stevens, \& Jacob, 2002) was presented over headphones using a laptop running PsyScope 1.2.5 (Cohen, MacWhinney, Flatt, \& Provost, 1993). In this test, participants indicated whether two 300-ms pure tones separated by a $100-\mathrm{ms}$ interval were the same or different in pitch by pressing the $S$ or $D$ key on the keyboard. The tone frequencies ranged from $325 \mathrm{~Hz}$ to $1994 \mathrm{~Hz}$; when the tones in a pair were different, the frequency ratios were $.67, .75, .85, .90$, or .95 . After 10 practice trials, 60 test trials were presented. The test trials consisted of 30 trials in which the tones in a pair were the same pitch (tone ratio $=1.0$ ) and 30 trials in which the tones were different, with each of the five frequency ratios occurring once per block of 10 trials. Trial types were randomly distributed within each block.

As in our prior studies (Bruder et al., 2004; Wexler et al., 1998), patients were separated into $\mathrm{D}(n=60)$ and $\mathrm{ND}(n=23)$ subgroups on the basis of their performance on this test. Patients were considered to be $\mathrm{D}$ if they made at most one error in 12 trials at the two easiest tone discrimination ratios (.67 and .75). The patients who did not pass this screening criterion were considered to be ND. Forty-seven of the 52 controls met this criterion, and only these participants were included in the control group. The original rationale given by Wexler et al. (1998) for using this criterion was to identify D patients, who have perceptual/ attentional competence in making simple tone discriminations (i.e., who like most healthy controls perform close to $100 \%$ correct at the easiest tone ratios), as opposed to ND patients, who fail to discriminate tones with clear pitch differences and may therefore have a basic deficit in auditory perception or attention. As is evident in Figure 1, the total percent correct scores (including tone ratios of $.67, .75, .85, .90, .95$, and 1.00 ) for participants in the current study show a bimodal negatively skewed distribution with two prominent maxima. The distribution of scores for D patients closely resembles that for healthy controls, with most having high accuracy levels at or above $90 \%$ correct. In contrast, ND patients showed markedly poorer performance, with a mean score $(69.5 \%$, $S D=8.4)$ more than two standard deviations below the mean for $\mathrm{D}$ patients $(M=93.4 \%, S D=7.9)$. This indicates that our screening criterion was successful in yielding two distinct subgroups of patients, one that performs as well as controls do in tone discrimination and one that shows a marked deficit. Furthermore, a cluster analysis of the tone discrimination scores for patients and controls (including accuracy scores for tone ratios of .67, .75, .85, $.90, .95$, and 1.00 as variables) yielded two clusters that show a close correspondence to the D and ND subgroups. Thus, $88.3 \%$ of the $\mathrm{D}$ patients fell in one cluster with high-accuracy scores, whereas $91.3 \%$ of the ND patients fell in the second cluster with low-accuracy scores. This further supports the use of our original screening criterion to define the D and ND subgroups.

WSPT. The WSPT (Wexler et al., 1998) was presented using the same equipment as in the tone discrimination task. Each trial began with four nouns spoken in a male voice, with $1 \mathrm{~s}$ between words. One of these words was then repeated after a delay of $9 \mathrm{~s}$. Participants were instructed to remember the four words in the order presented and to indicate the position of the repeated word by pressing the $1,2,3$, or 4 key on the keyboard. The WSPT consisted of 36 trials, randomly ordered and balanced with regard to the four serial positions. No word appeared twice in the test.

Neuropsychological tests. Most patients $(n=78)$ and controls $(n=51)$ were also tested on the Letter-Number Sequencing Test from the Wechsler Adult Intelligence Scale-Third Edition (WAIS-III; Tulsky, Zhu, \& Ledbetter, 1997; Wechsler, 1997) and the CPT-IP test (Cornblatt \& Keilp, 1994). The letter-number WM test consists of auditory presentation of strings of intermingled letters and numbers, and participants are asked to store and reorder the numbers and letters (i.e., recite in numeric and alphabetical order). The dependent measure is the total number of correct strings. Sustained attention was assessed with the four-digit fast

\footnotetext{
${ }^{2}$ Group differences reported below on the tone discrimination, WSPT, Letter-Number Sequence Test, CPT-IP, and WMS-R remained the same when we excluded the 13 patients who did not have a DIGS interview.

${ }^{3}$ To check whether nonpayment of inpatients may have impacted their incentive to perform the tests, we compared their performance with that of outpatients (who were paid) on the tone discrimination test and the WSPT. There was no significant difference between inpatients $(n=56)$ and outpatients $(n=27)$ in their tone discrimination, $F(1,81)=0.04, p=.85$, or WSPT performance, $F(1,81)=0.34, p=.56$. It is therefore unlikely that nonpayment of inpatients differentially affected their performance.
} 


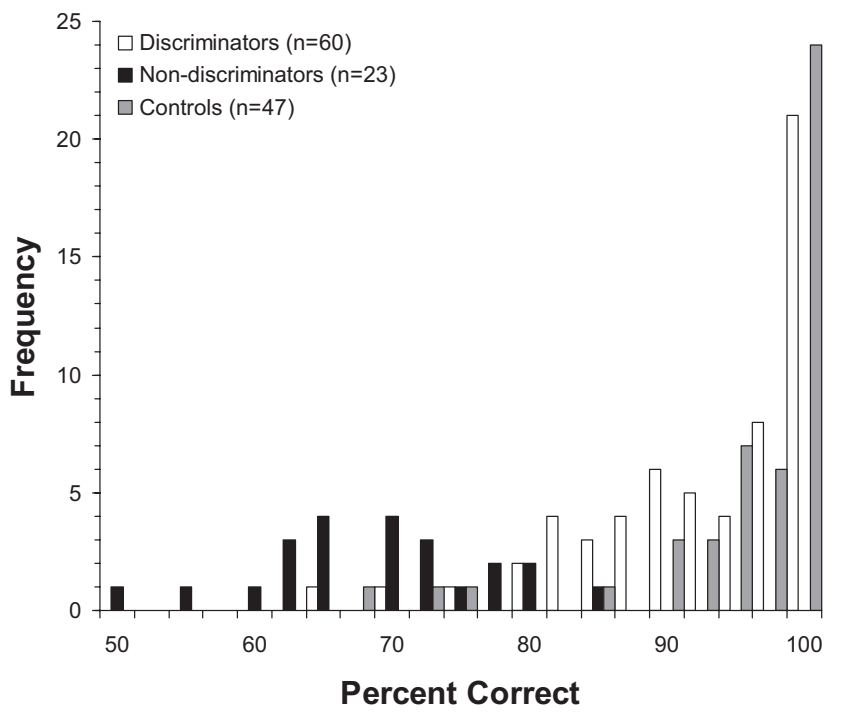

Figure 1. Distribution of percentage correct on the tone discrimination test for discriminators, nondiscriminators, and controls. The lower and upper limits for percentage correct intervals are $50 \leq X<52.5,52.5 \leq$ $X<55,55 \leq X<57.5,57.5 \leq X<60,60 \leq X<62.5, \ldots X=100$.

condition of the CPT-IP test (Cornblatt, Risch, Faris, Friedman, \& Erlenmeyer-Kimling, 1988). Number strings were presented on a Macintosh laptop screen for $50 \mathrm{~ms}$ at a constant rate of 1 per second. Participants responded with a key press if the number string matched the string that had preceded it (i.e., same digits in same order). A total of 150 stimuli were presented: 28 target trials, 25 catch trials, and 97 random trials. Performance on the CPT-IP was measured by (a) correct detections or hits (responses to target trials), (b) false alarms (responses to catch trials), and (c) $d^{\prime}$ index of sensitivity computed from hits and false alarms using a signaldetection computer program (Cornblatt et al., 1988). Verbal and performance IQ scores on the WAIS-III (Wechsler, 1997) were also obtained for 47 patients, and WMS-R indices of verbal and visual memory (Wechsler, 1987) were obtained for 50 patients as part of other ongoing research at the New York State Psychiatric Institute.

\section{Statistical Analyses}

Comparison of age, education, and handedness (Edinburgh Inventory laterality quotient; Oldfield, 1971) between patient and control groups was performed using a one-way analysis of variance (ANOVA) followed by Student Newman-Keuls (SNK) pairwise comparisons. Gender was compared between groups with a chi-square test. The influence of medication on the percentage of correct responses in the tone discrimination test was first analyzed using a Group (unmedicated patients, medicated patients, controls) $\times$ Gender (male, female) $\times$ Ratio of Tones $(.67, .75, .85$, $.90, .95,1.00)$ repeated measures ANOVA. Similarly, accuracy scores on the WSPT were submitted to a Group (unmedicated patients, medicated patients, controls) $\times$ Gender (male, female) $\times$ Serial Position $(1,2,3,4)$ repeated measures ANOVA. Accuracy scores on the WSPT were also submitted to a Group (D, ND, controls $) \times$ Gender $($ male, female $) \times$ Serial Position $(1,2,3,4)$ repeated measures ANOVA followed by SNK comparisons. Performance on the Letter-Number Sequencing and CPT-IP tests was analyzed using a Group $(\mathrm{D}, \mathrm{ND}$, controls) $\times$ Gender (male, female) ANOVA. Main effects of group were followed by SNK multiple comparisons, and significant interactions were followed by simple effects analyses and pairwise contrasts using the BMDP-4V program (Dixon, 1992). Greenhouse-Geisser epsilon ( $\varepsilon$ ) correction was used to compensate for violations of sphericity when appropriate (see e.g., Keselman, 1998). Eta-squared $\left(\eta^{2}\right)$ and Cohen's $d$ measures of effect size are also presented. A conventional significance level $(p<.05)$ was applied for all effects.

To examine the impact of auditory hallucinations in patients having a verbal WM deficit, we separated patients in the D subgroup into those who reported experiencing auditory hallucinations in the past week (a rating of one or higher on an auditory hallucination item of the SAPS; $n=18$ ) and those who did not report auditory hallucinations $(n=33)$. ANOVAs comparing hallucinators, nonhallucinators, and controls were performed using the same statistical analyses as for the $\mathrm{D}, \mathrm{ND}$, and control groups.

The relationships between the total accuracy score on the WSPT and age or education were measured with Pearson correlations. Correlations also examined relationships between the total WSPT scores and ratings of auditory hallucinations on the SAPS, which were available for $51 \mathrm{D}$ patients and $19 \mathrm{ND}$ patients. These correlations with WSPT were also performed for positive and negative symptom total scores on the PANSS, available for $53 \mathrm{D}$ patients and 19 ND patients. Correlations also examined relationships between WSPT accuracy and performance on the tone discrimination, LetterNumber Sequencing, CPT-IP, and WMS-R tests.

\section{Results}

\section{Unmedicated Patients, Medicated Patients, and Healthy Controls}

A comparison was made of tone discrimination and WSPT performance for 38 unmedicated patients ( 24 male), 45 medicated patients (25 male), and 47 healthy controls ( 23 male). There was no difference between medicated patients and unmedicated patients in age, education, or handedness, but both patient groups were somewhat less educated than controls were (see Table 1). Unmedicated patients were somewhat older than controls, but medicated patients were not significantly different in age from either group. Performance on the WSPT was not significantly correlated with age, $r(47)=-.10, n s$, or education, $r(47)=.24, n s$, in controls and only weakly correlated with age, $r(83)=-.27, p<$ .05 , and education, $r(83)=.22, p<.05$, in patients.

The ANOVA of the tone discrimination performance of unmedicated patients, medicated patients, and controls revealed significant main effects of group, $F(2,124)=7.90, p=.001, \eta^{2}=$ .11 , and tone ratio, $F(5,620)=23.92 p<.001, \varepsilon=0.515, \eta^{2}=$ .16. As can be seen in Table 1, the larger the difference in pitch between tone pairs, where a tone ratio of .95 is the smallest and .67 is the largest difference, the greater the accuracy of tone discrimination. Also, healthy controls performed more accurately than did the patient groups on the tone discrimination test $(p<.05)$, but there was no difference between unmedicated and medicated patients. The ANOVA of WSPT performance revealed main effects of group, $F(2,124)=20.47, p<.001, \eta^{2}=.25$, and serial 
Table 1

Demographics and Mean Percent Correct in the Tone Discrimination Test and WSPT for Unmedicated Patients, Medicated Patients, and Healthy Controls

\begin{tabular}{lcccc}
\hline \multicolumn{1}{c}{ Variable } & Unmedicated & Medicated & Control & Statistics \\
\hline Age (years) & $31.4_{\mathrm{a}}(10.9)$ & $29.9_{\mathrm{a} . \mathrm{b}}(7.4)$ & $26.6_{\mathrm{b}}(6.6)$ & $F(2,127)=3.70^{*}$ \\
Education (years) & $13.9_{\mathrm{a}}(2.3)$ & $14.3_{\mathrm{a}}(2.7)$ & $16 . \mathrm{b}_{\mathrm{b}}(2.0)$ & $F(2,127)=12.31^{* * * *}$ \\
Handedness (LQ) & $65.2(41.5)$ & $74.9(33.4)$ & $77.2(19.25)$ & $F(2,127)=1.61$ \\
Tone ratio (\%) & & & & \\
.67 & $88.16(17.72)$ & $89.26(16.73)$ & $98.23(5.19)$ & \\
.75 & $89.04(19.48)$ & $89.63(16.78)$ & $99.29(3.40)$ & \\
.85 & $84.65(23.69)$ & $81.85(26.31)$ & $94.68(13.51)$ & \\
.90 & $69.30(36.25)$ & $78.89(25.48)$ & $91.13(18.99)$ & \\
.95 & $72.37(32.25)$ & $71.85(36.21)$ & $89.36(22.64)$ & $F(2,124)=7.90^{* * *}$ \\
Total correct & $83.27 \mathrm{a}(19.72)$ & $83.32_{\mathrm{a}}(17.44)$ & $95.09 \mathrm{~b}(9.25)$ & \\
WSPT position $(\%)$ & & & & \\
1 & & & & \\
2 & $81.14(19.44)$ & $81.85(22.70)$ & $95.04(9.14)$ & \\
3 & $73.25(24.67)$ & $72.22(24.62)$ & $95.04(10.94)$ & \\
4 & $65.79(27.11)$ & $68.89(29.22)$ & $87.59(15.72)$ & \\
Total correct & $89.47(19.15)$ & $83.33(18.12)$ & $98.58(5.85)$ & $F(2,124)=20.47^{* * * * *}$ \\
\hline
\end{tabular}

Note. Unmedicated patients: $n=38$ (24 male); medicated patients: $n=45$ ( 25 male); controls: $n=47$ ( 23 male). Standard deviations are given in parentheses. Means with different subscripts differ significantly at $p<$ .05 using Student Newman-Keuls post hoc comparisons. WSPT $=$ Word Serial Position Test; LQ = laterality quotient on the Edinburgh Inventory. ${ }^{*} p<.05 . \quad{ }^{* *} p<.01 .{ }^{* * * *} p<.001$.

position, $F(3,372)=23.78, p<.001, \varepsilon=0.89, \eta^{2}=.16$. The controls showed overall greater accuracy than did the patient groups on the WSPT $(p<.05)$. Most importantly, there was no difference in WSPT performance between unmedicated and medicated patients, which indicates that there was no evidence of medication effects on this verbal WM test. Neither the gender main effect nor any interaction involving gender was significant for either the WSPT or tone discrimination test.

\section{Discriminators, Nondiscriminators, and Healthy Controls}

Table 2 gives the demographic variables for the $\mathrm{D}, \mathrm{ND}$, and control groups. There was no difference in gender, age, or education between the D and ND groups, but these groups were somewhat older and less educated than were controls. There was no difference between groups in handedness. An approximately equal percentage of D patients (46.7\%) and ND patients (43.5\%) were off antipsychotic medication when tested, and the remainder of the patients in each group were receiving atypical antipsychotics. The $\mathrm{D}$ and ND patients did not differ in age of onset or illness duration. There was no difference between the patient groups in overall symptom severity as indexed by total BPRS scores.

The ANOVA of the WSPT performance of $\mathrm{D}, \mathrm{ND}$, and controls revealed significant main effects of group, $F(2,124)=53.20, p<$ $.001, \eta^{2}=.46$, and serial position, $F(3,372)=20.61, p<.001$, $\varepsilon=0.893, \eta^{2}=.14$, but no significant Group $\times$ Serial Position interaction. ${ }^{4}$ Multiple comparisons indicated that ND patients had overall poorer accuracy than did D patients (Cohen's $d=1.44$ ) and controls $(d=2.73)$ on the WSPT $(p<.05)$ and that D patients also had significantly poorer accuracy than did controls $(d=1.08$, $p<.05)$. Neither the gender main effect nor any interaction involving gender was significant. Figure 2 shows the mean accuracy for each group at the four serial positions on the WSPT in both the current study and our prior study (Bruder et al., 2004). ND patients performed considerably worse than did both $\mathrm{D}$ patients and controls at all serial positions. D patients showed smaller deficits compared with controls for words in Positions 1-3, and their accuracy for the fourth word in the sequence (92\% correct) approached that for controls. WSPT performance was not significantly correlated with tone discrimination accuracy in controls, $r(47)=.22, n s ; \mathrm{D}$ patients, $r(60)=-.01, n s$; or ND patients, $r(23)=-.29, n s$.

Ratings of symptom severity on the PANSS were available for $53 \mathrm{D}$ patients and $19 \mathrm{ND}$ patients. There was a trend for ND patients to have the expected higher negative symptoms when compared with D patients (see Table 2), but there was no significant difference between these groups in either the PANSS positive or negative symptom total scores.

\section{Auditory Hallucinators, Nonhallucinators, and Controls}

To examine whether the verbal WM deficit in D patients is greater for those who are prone to auditory hallucinations, we compared the WSPT performance for $18 \mathrm{D}$ patients (10 male) who reported experiencing auditory hallucinations in the past week (a rating of one or higher on an auditory hallucination item of the SAPS), 33 D patients (18 male) without auditory hallucinations,

\footnotetext{
${ }^{4}$ Differences in WSPT performance between the D, ND, and control groups remained the same when the 23 patients having a diagnosis of schizoaffective disorder were excluded from the analyses. The ANOVA revealed the same main effect of group, $\mathrm{F}(2,101)=43.61, p<.001$, but no Group $\times$ Serial Position interaction. Multiple comparisons indicated that ND patients $(n=18)$ had overall poorer accuracy than did D patients $(n=42)$ and controls $(p<.05)$, and D patients had poorer accuracy than did controls $(p<.05)$.
} 
Table 2

Means (and Standard Deviations) for Demographic, Clinical, and Neuropsychological Variables for Discriminators (Ds), Nondiscriminators (NDs), and Controls

\begin{tabular}{lcccl}
\hline \multicolumn{1}{c}{ Variable } & $\mathrm{D}$ & $\mathrm{ND}$ & Control & \multicolumn{1}{c}{ Statistics } \\
\hline Age (years) & $30.6(8.8)$ & $30.6(10.3)$ & $26.6(6.5)$ & $F(2,127)=3.33^{*}$ \\
Education (years) & $14.4_{\mathrm{a}}(2.5)$ & $13.5 \mathrm{a}(2.5)$ & $16.3_{\mathrm{b}}(2.1)$ & $F(2,127)=13.47^{* * * *}$ \\
Handedness (LQ) & $69.9(39.1)$ & $71.8(33.3)$ & $73.7(28.7)$ & $F(2,127)=0.69$ \\
Onset age (years) & $22.1(6.8)$ & $22.1(6.7)$ & & $t(81)=0.03$ \\
Illness duration (years) & $8.5(8.2)$ & $8.5(9.6)$ & & $t(81)=0.01$ \\
Total BPRS & $35.9(13.3)$ & $35.6(10.8)$ & & $t(70)=0.08$ \\
PANSS & & & \\
$\quad$ Positive & $15.0(7.1)$ & $14.2(5.7)$ & & $t(70)=0.43$ \\
$\quad$ Negative & $14.3(6.0)$ & $16.6(5.9)$ & & $t(70)=1.44$ \\
Letter-Number Sequencing Test ${ }^{\mathrm{b}}$ & $10.6_{\mathrm{a}}(3.0)$ & $8.3_{\mathrm{b}}(2.9)$ & $12.5_{\mathrm{c}}(2.8)$ & $F(2,122)=15.44^{* * * * *}$ \\
$\quad($ no. correct) & $1.75_{\mathrm{a}}(0.99)$ & $0.98_{\mathrm{b}}(0.66)$ & $2.59_{\mathrm{c}}(0.81)$ & $F(2,122)=25.60^{* * * *}$ \\
CPT-IP $\left(d^{\prime}\right)$ & & &
\end{tabular}

Note. Ds: $n=60$ (35 male); NDs: $n=23$ (14 male); controls: $n=47$ (23 male). Means with different subscripts differ significantly at $p<.05$ using Student Newman-Keuls post hoc comparisons. LQ $=$ laterality quotient on the Edinburgh Inventory; BPRS = Brief Psychiatric Rating Scale; PANSS = Positive and Negative Symptom Scale; CPT-IP = Continuous Performance Test-Identical Pairs.

a Ds: $n=53$; NDs: $n=19$. ${ }^{\text {b Ds: }} n=59$; NDs: $n=22$; controls: $n=47$.

${ }^{*} p<.05$. **** $p<.001$.

and the 47 healthy controls (23 male). There was no significant difference between hallucinators and nonhallucinators in gender, age, education, or handedness, but hallucinators $(M=14.4$ years, $S D=2.4)$ and nonhallucinators $(M=14.5$ years, $S D=2.5)$ were somewhat less educated than controls $(M=16.3$ years, $S D=2.0)$, $F(2,97)=7.70, p=.001$. As expected, there was also no difference in tone discrimination performance between $\mathrm{D}$ patients having hallucinations, D patients without hallucinations, and controls.

An ANOVA revealed significant main effects of group, $F(2$, $92)=19.01, p<.001, \eta^{2}=.29$, and serial position, $F(3,276)=$ 24.36, $p<.001, \varepsilon=0.89, \eta^{2}=.21$, and a Group $\times$ Serial Position interaction, $F(6,276)=3.18, p<.01, \varepsilon=0.89, \eta^{2}=.06$. Although analysis of simple effects yielded significant group differences at each serial position, group differences at the second and third serial positions, $F(2,92) \geq 9.84, p<.001$, were more marked than those at the first and fourth serial positions, $F(2$,
92 ) $\geq 6.59, p<.01$ (see Figure 3). Pairwise comparisons indicated that hallucinators had significantly poorer accuracy than did controls at all serial positions, $F(1,92) \geq 7.33, p<.01, d=0.94$ to 1.44. Nonhallucinators showed significantly poorer accuracy than did controls at Serial Positions 1, 2, and 4, F(1, 92) $\geq 7.08, p<$ $.01, d=0.76$ to .93 , but not at the third serial position $(d=0.43)$. Most importantly, hallucinators performed more poorly than did nonhallucinators only at the middle Serial Positions 2 and 3, $F(1$, $92) \geq 5.04, p<.05, d=0.54$ and 0.74 . There was no significant effect of or interactions involving gender.

\section{Correlations of WSPT Scores and Symptom Ratings}

To examine whether the verbal WM deficit in D patients was correlated with auditory hallucinations, we correlated their overall accuracy scores on the WSPT with ratings on the SAPS. Poorer performance significantly correlated with greater auditory halluci-
Bruder et al (2004)

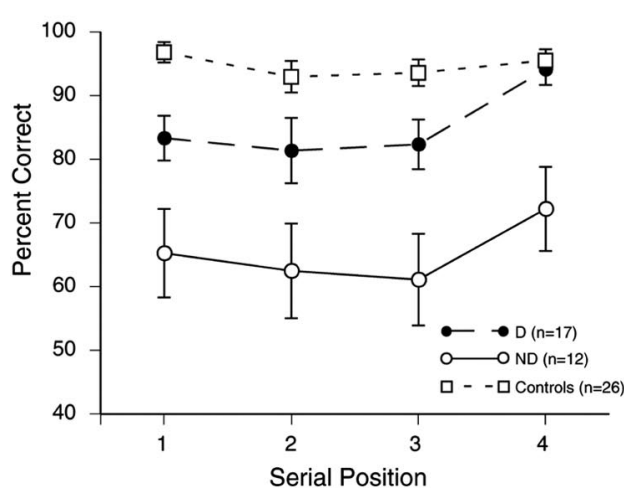

Current Study

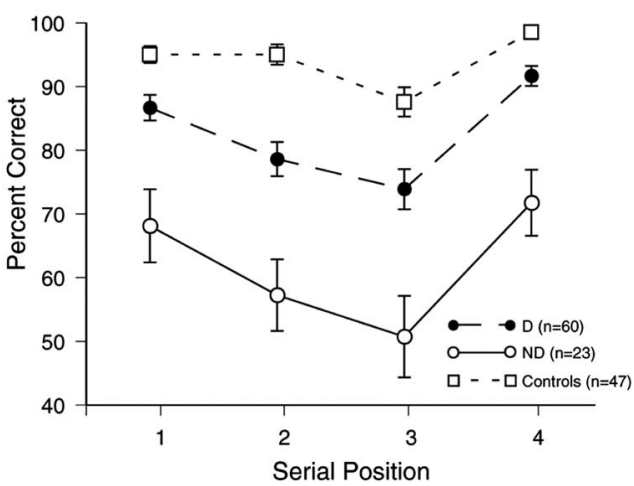

Figure 2. Mean percentage of correct responses for discriminators (Ds), nondiscriminators (NDs), and controls as a function of the serial position of words on the Word Serial Position Test for the Bruder et al. (2004) and current studies. Error bars indicate standard errors of the mean. 

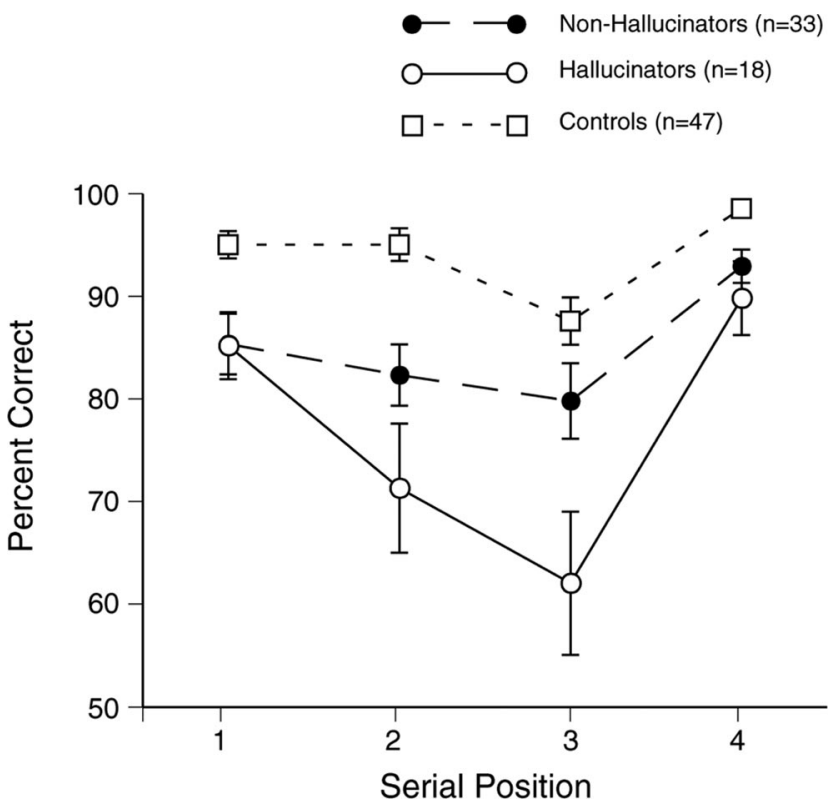

Figure 3. Mean percentage of correct responses for hallucinators, nonhallucinators, and controls as a function of the serial position of words on the Word Serial Position Test. Error bars indicate standard errors of the mean.

nation ratings, $r(51)=-.35, p=.01$. Hallucinatory behavior ratings in D patients on the PANSS were also significantly correlated with poorer WSPT performance, $r(53)=-.27, p<.05$. There were, however, no significant correlations between WSPT scores and either PANSS total positive or negative symptom ratings for D patients, $r(53) \leq .18, n s$. WSPT performance in ND patients was not correlated with hallucination ratings on the SAPS, $r(19)=.02, n s$, or PANSS, $r(19)=.04, n s$. Nor were there significant correlations of performance on the Letter-Number Sequencing or CPT-IP tests and symptom ratings in either D or ND patients (all $p \mathrm{~s}>.14$ ).

\section{Neuropsychological Tests}

There was a significant difference between the D, ND, and control groups in their performance on the Letter-Number Sequencing Test, $F(2,122)=15.44, p<.001, \eta^{2}=.20$. As shown in Table 2, ND patients performed more poorly than did both D patients and controls $(p<.05)$. Although $\mathrm{D}$ patients also differed significantly from controls $(d=0.60)$, their deficit was smaller than that for ND patients $(d=1.41)$. There was no significant gender effect. The findings for this auditory WM test confirm the group differences seen on the WSPT. The letter-number test was significantly correlated with performance on the WSPT in D patients, $r(59)=.47, p<.001$; ND patients, $r(22)=.52, p=.01$, and controls, $r(47)=.44, p<.01$.

ND patients also performed significantly worse than did D patients and controls on the CPT-IP, $F(2,122)=25.60, p<.001$, $\eta^{2}=.30$ (see Table 2). Their deficit was on the average more than two standard deviations below the mean for controls $(d=2.18)$, which is consistent with a marked deficit in sustained attention. D patients showed a more moderate deficit in CPT-IP performance compared with controls $(d=0.93)$. There was no significant gender effect. The CPT-IP test was not significantly correlated with performance on the WSPT in D patients, $r(59)=.22, n s$; ND patients, $r(22)=.31, n s$; or controls, $r(52)=.24$, $n s$.

ND patients had lower verbal and performance IQ scores on the WAIS-III than did D patients, in keeping with a generalized cognitive deficit, whereas D patients had IQs in the normal range (see Table 3). On the WMS-R, however, D patients performed as poorly as did ND patients on the verbal memory index but tended to show better visual memory. D patients performed significantly poorer on the verbal than visual memory index, $t(38)=4.36, p<$ .001 , whereas ND patients did not show a difference between their verbal and visual memory, $t(10)=0.83, n s$. The WMS-R indices were standardized to have a mean of 100 and a standard deviation of 15. The WMS-R scores for D and ND patients with an education level of 14.4 and 13.5 years, respectively, were compared with norms of the standardization sample with an education level greater than 12 years (Wechsler, 1987). The verbal memory index for $\mathrm{D}$ patients was between one and two standard deviations below the mean for the standardization sample $(M=107.6, S D=14.7)$, whereas their visual memory index was within a half a standard deviation of the norms $(M=105.5, S D=13.4)$. The ND patients showed deficits in both verbal and visual memory, which were between one and two standard deviations below the norms. D and ND patients also showed a different pattern of correlations between performance on the WMS-R and the WSPT. Among D patients, performance on the WSPT was associated with the verbal memory index on the WMS-R, $r(39)=.32, p=.05$, with a significant correlation only for words in the third serial position, $r(39)=.41, p<.01$, but was not correlated with the visual memory index, $r(40)=.18, n s$. In ND patients, performance on the WSPT was strongly correlated with the visual memory index on the WMS-R, $r(11)=.75, p<.01$, with a significant correlation only for words in the second serial position, $r(11)=.72, p=.01$, but was not correlated with the verbal memory index on the WMS-R, $r(11)=.16, n s$.

\section{Discussion}

Patients with schizophrenia having normal performance on a tone discrimination test of auditory perception and attention (D patients) had deficits in verbal WM on the WSPT, which replicates

Table 3

Means (and Standard Deviations) for Neuropsychological Measures for Verbal and Nonverbal Tests

\begin{tabular}{lccc}
\hline \multicolumn{1}{c}{ Measure } & D & ND & Statistics \\
\hline WAIS-III $^{\mathrm{a}}$ & & & \\
$\quad$ Verbal IQ & $104.3(14.6)$ & $88.1(15.8)$ & $t(45)=3.23^{* * *}$ \\
$\quad$ Performance IQ & $95.8(15.1)$ & $79.8(21.2)$ & $t(45)=2.84^{* *}$ \\
WMS-R & & & \\
$\quad$ Verbal Memory & $85.6(18.1)$ & $82.9(12.4)$ & $t(48)=0.46$ \\
$\quad$ Visual Memory & $99.3(19.8)$ & $88.5(21.0)$ & $t(48)=1.57$ \\
\hline
\end{tabular}

Note. $\mathrm{D}=$ discriminator; $\mathrm{ND}=$ nondiscriminator; WAIS-III $=$ Wechsler Adult Intelligence Scale-Third Edition; WMS-R = Wechsler Memory Scale-Revised.

${ }^{\mathrm{a}}$ Ds: $n=35$; NDs: $n=12 . \quad{ }^{\mathrm{b}}$ Ds: $n=39$; NDs: $n=11$.

***: $p<.01$. 
our prior findings (Bruder et al., 2004; Wexler, Donegan, Stevens, \& Jacob, 2002; Wexler et al., 1998). These patients also showed deficits on another test of auditory verbal WM (i.e., the LetterNumber Sequencing Test) and on the WMS-R index of verbal explicit memory, but their nonverbal performance was relatively preserved. As in our prior study (Bruder et al., 2004), their verbal memory scores on the WMS-R were one to two standard deviations below published norms, a large effect size (Cohen, 1988), but they showed little or no deficit on the visual memory index. The present study included inpatients and outpatients, generating a large sample with a range of symptoms and symptom severity. We were therefore able to demonstrate for the first time associations between the verbal WM deficit in D patients and symptoms of auditory hallucinations. In addition, $46 \%$ of patients were evaluated while off antipsychotic medications, making it possible to show that there were no differences between medicated and unmedicated patients on the tone discrimination test that defined the subgroups or on the WSPT that revealed the verbal WM deficit in D patients.

Patients who failed the tone discrimination test (i.e., NDs) performed more poorly than did D patients and controls on the WSPT and on the Letter-Number Sequencing Test measuring auditory WM. They also showed poor verbal and visual memory on the WMS-R, which agrees with prior findings of both verbal and nonverbal memory deficits in this subgroup (Bruder et al., 2004; Wexler et al., 1998). One interpretation of the ND patients' poor performance on the tone discrimination test and on the WSPT is that they have a basic deficit in auditory processing. This possibility receives some support from the findings of Javitt, Strous, Grochowski, Ritter, and Cowan (1997), who compared the tone-matching performance of patients with schizophrenia and healthy controls. Patients showed deficits in the ability to match two tones in both easy and difficult pitch discriminations, even when the interval between tones was brief $(\leq 1 \mathrm{~s})$. They suggested that this deficit in auditory sensory or echoic processing reflects impaired precision with which patients with schizophrenia encode the physical properties of auditory stimuli. We found that both unmedicated and medicated patients with schizophrenia showed a deficit in tone discrimination, but this deficit was present in only a subgroup of patients, whom we refer to as ND patients. Although their poor performance on the WM tests may stem from an auditory processing deficit, it might also reflect a generalized cognitive dysfunction that cuts across modality. Their marked deficit on the CPT-IP and on the visual memory index of the WMS-R is suggestive of a more global problem, which may in part involve reduced sustained attention to both auditory and visual stimuli.

The normal tone discrimination and nonverbal memory in D patients indicates that they do not suffer from a generalized cognitive deficit and that their poor verbal WM cannot be explained by nonspecific factors, such as a lack of attention or perceptual dysfunction. Neuroimaging studies have suggested that a neural network involving the prefrontal and parietal regions underlies WM performance (Goldman-Rakic, 1991; Smith \& Jonides, 1999). Healthy adults were found to activate the left inferior frontal cortex, temporal cortex, and left inferior and superior parietal lobes during performance of the WSPT (Stevens, Goldman-Rakic, Gore, Fulbright, \& Wexler, 1998). Activation of the left inferior frontal cortex was reduced in patients with schizophrenia who performed at least $90 \%$ correct on the tone discrimination test (i.e., D patients), and they also failed to show greater activation of this region during a word test than during a tone serial position test, which was seen in healthy adults (Stevens et al., 1998). Similarly, Barch et al. (2002) measured fMRI during both WM (n-back) and recognition memory tasks with words and unfamiliar faces and found that patients with schizophrenia failed to show greater activation for words than faces in regions that normally show enhanced activation to verbal stimuli, including left inferior prefrontal, left parietal, and left temporal cortex. Recently, Kayser et al. (2010) recorded event-related brain potentials (ERPs) of patients with schizophrenia and controls during recognition memory tasks with words and unfamiliar faces. Old-new ERP effects were markedly reduced in patients over the left lateral parietotemporal region, and this deficit was more pronounced for words than faces despite the greater difficulty in recalling faces, which indicates that it was not due to a generalized deficit. Similarly, in a study recording ERPs of patients with schizophrenia during the WSPT, Kayser et al. (2006) found evidence of disturbed processing in a frontalparietotemporal network during encoding and early storage of the words. These findings suggest that both verbal WM and explicit memory deficits in schizophrenia may reflect a common disturbance of frontal and left parietotemporal regions. This is consistent with our findings for D patients, who showed poorer verbal memory not only on WM tests but also on the WMS-R.

Overall severity of clinical symptoms could not account for the marked difference in performance of the D and ND subgroups on the verbal WM tests. ND patients in our prior study did show somewhat higher negative symptoms than did D patients on the PANSS (Bruder et al., 2004), but this difference was smaller and not statistically significant in the current study. Although it could be argued that higher negative symptoms and possible reduced motivation or effort might be related to the generally poorer performance in ND patients, severity of negative symptoms was not correlated with performance on the WSPT.

Poorer verbal WM on the WSPT in D patients, but not in ND patients, was significantly correlated with auditory hallucination ratings on the SAPS and hallucination ratings on the PANSS but not with negative symptoms. As predicted on the basis of fMRI findings of reduced activity in language-related cortical regions in patients with auditory hallucinations (Wible et al., 2009), D patients having auditory hallucinations showed poorer WSPT performance than did those without hallucinations and healthy adults. Although this could be due to hallucinations' interfering with auditory processing of words, it is important to note that hallucinators performed more poorly than did nonhallucinators only at the middle serial positions on the WSPT. An alternative interpretation is that patients with auditory hallucinations may be more prone to cognitive sources of interference (e.g., proactive and retroactive interference; Stevens et al., 2000). The relation between poorer WSPT performance and auditory hallucinations is consistent with the hypothesis that verbal WM deficits in schizophrenia stem from dysfunction of language-related regions in the left inferior prefrontal and parietotemporal cortex (Stevens et al., 1998; Wible et al., 2009).

The importance of our findings stems from the need to parse the heterogeneous clinically diagnosed disorder of schizophrenia into subgroups having more homogeneous pathophysiology. The tone discrimination test introduced by Wexler et al. (1998) provides a 
quick and inexpensive way of identifying patients who have marked deficits in auditory perception or attention and display widespread cognitive dysfunction on both verbal and nonverbal tests. These ND patients differ from those who have normal tone discrimination (i.e., D patients) in showing poorer performance on auditory verbal WM tests (the WSPT and the Letter-Number Sequencing Test), poorer sustained attention (CPT-IP), and lower verbal and performance IQ. This subgroup therefore has global cognitive deficits as might result from perceptual or attentional dysfunction and differs from D patients, who have a more focal deficit in verbal memory. Deficits in early perceptual processing could have downstream impact on higher order cognitive or social functions (Javitt et al., 1997; Wynn, Sugar, Horan, Kern, \& Green, 2010). A cognitive rehabilitation strategy using auditory training was found to improve verbal WM in schizophrenia (Fisher, Holland, Merzenich, \& Vinogradov, 2009) and may prove particularly beneficial for ND patients having an auditory processing deficit.

This study has several limitations. First, the tests included in this study were not sufficiently broad for determining the specificity of the verbal WM deficit in D patients. Wexler et al. (1998) and Stevens et al. (2000) did, however, show that D patients had deficits on the WSPT but not on a tone serial position test, which supports the specificity of their verbal WM deficit. Also, Wexler et al. (2002) found that D patients had marked deficits on a serial position test with easily named environmental sounds (e.g., telephone ringing) but performed nearly as well as healthy controls on the same test with birdsongs that could not be verbally labeled and were much more difficult for controls. Second, it is not clear whether the poor performance in ND patients on the verbal WM tests was due to an auditory processing deficit or more global attentional dysfunction. This could be addressed by measuring early auditory ERPs (N1, P2) during the WSPT and also mismatch negativity to assess preattentive auditory processing. Third, whereas D patients have been found to show reduced verbal WM on both auditory and visual versions of the WSPT (Stevens et al., 2000), the extent to which the deficits in ND patients are specific to the auditory modality needs further study. Last, the D subgroup included $60 \%-70 \%$ of patients with schizophrenia in our studies and was defined by their "normal" performance in one cognitive task. This raises a question as to whether they represent a homogeneous subtype of schizophrenia or could benefit from further subdivision on the basis of their clinical features (e.g., those with or without auditory hallucinations) or distinctive cognitive or neurophysiologic deficits.

\section{References}

American Psychiatric Association. (2000). Diagnostic and statistical manual of mental disorders (4th ed., text rev.). Washington, DC: Author.

Andreasen, N. C. (1983). The Scale for the Assessment of Negative Symptoms (SANS). Iowa City, IA: University of Iowa.

Andreasen, N. C. (1984). The Scale for the Assessment of Positive Symptoms (SAPS). Iowa City, IA: University of Iowa.

Barch, D. M., Csernansky, J. G., Conturo, T., \& Snyder, A. Z. (2002). Working and long-term memory deficits in schizophrenia: Is there a common prefrontal mechanism? Journal of Abnormal Psychology, 111, 478-494. doi:10.1037/0021-843X.111.3.478

Bruder, G. E., Wexler, B. E., Sage, M. M., Gil, R. B., \& Gorman, J. M. (2004). Verbal memory in schizophrenia: Additional evidence of subtypes having different cognitive deficits. Schizophrenia Research, 68, 137-147. doi:10.1016/S0920-9964(03)00156-7
Callicott, J. H., Bertolino, A., Mattay, V. S., Langheim, F. J., Duyn, J., Coppola, R., ... Weinberger, D. R. (2000). Physiological dysfunction of the dorsolateral prefrontal cortex in schizophrenia revisited. Cerebral Cortex, 10, 1078-1092. doi:10.1093/cercor/10.11.1078

Carter, C. S., Braver, T. S., Barch, D. M., Botvinick, M. M., Noll, D., \& Cohen, J. D. (1998, May 1). Anterior cingulate cortex, error detection, and the online monitoring of performance. Science, 280, 747-749. doi:10.1126/science.280.5364.747

Carter, C. S., Robertson, L. C., Nordahl, T. E., Chaderjian, M., \& OshoraCelaya, L. (1996). Perceptual and attentional asymmetries in schizophrenia: Further evidence for a left hemisphere deficit. Psychiatry Research, 62, 111-119. doi:10.1016/0165-1781(96)02849-1

Cohen, J. (1988). Statistical power analysis for behavioral sciences (2nd ed.). Hillside, NJ: Erlbaum.

Cohen, J. D., MacWhinney, B., Flatt, M., \& Provost, J. (1993). PsyScope: A new graphic interactive environment for designing psychology experiments. Behavioral Research Methods, Instruments \& Computers, 25 , 257-271.

Cornblatt, B. A., \& Keilp, J. G. (1994). Impaired attention, genetics, and the pathophysiology of schizophrenia. Schizophrenia Bulletin, 20, 31-46.

Cornblatt, B. A., Risch, N. J., Faris, G., Friedman, D., \& ErlenmeyerKimling, L. (1988). The Continuous Performance Test, Identical Pairs version (CPT-IP): I. New findings about sustained attention in normal families. Psychiatry Research, 26, 223-238. doi:10.1016/01651781(88)90076-5

Dixon, W. J. (Ed.). (1992). BMDP statistical software manual: To accompany the 7.0 software release. Berkeley, CA: University of California Press.

Donohoe, G., Corvin, A., \& Robertson, I. H. (2006). Evidence that specific executive functions predict symptom variance among schizophrenia patients with a predominantly negative symptom profile. Cognitive Neuropsychiatry, 11, 13-32. doi:10.1080/13546800444000155

Egan, M. F., Goldberg, T. E., Gscheidle, T., Weirich, M., Rawlings, R., Hyde, T. M., ... Weinberger, D. R. (2001). Relative risk for cognitive impairments in siblings of patients with schizophrenia. Biological Psychiatry, 50, 98-107. doi:10.1016/S0006-3223(01)01133-7

Endicott, J., \& Spitzer, R. L. (1978). A diagnostic interview: The schedule for affective disorders and schizophrenia. Archives of General Psychiatry, 35, 837-844.

First, M. B., Spitzer, R. L., Gibbon, M., \& Williams, J. B. W. (1996). Structured Clinical Interview for DSM-IV Axis I Disorders: Non-patient edition (SCID-NP). New York, NY: Biometrics Research Department, New York State Psychiatric Institute.

First, M. B., Spitzer, R. L., Gibbon, M., \& Williams, J. B. W. (2002). Structured Clinical Interview for DSM-IV-TR Axis I Disorders: Research version: Patient edition (SCID-I/P). New York, NY: Biometrics Research Department, New York State Psychiatric Institute.

Fisher, M., Holland, C., Merzenich, M. M., \& Vinogradov, S. (2009). Using neuroplasticity-based auditory training to improve verbal memory in schizophrenia. American Journal of Psychiatry, 166, 805-811. doi: 10.1176/appi.ajp.2009.08050757

Gold, J. M., Carpenter, C., Randolph, C., Goldberg, T. E., \& Weinberger, D. R. (1997). Auditory working memory and Wisconsin Card Sorting Test performance in schizophrenia. Archives of General Psychiatry, 54, 159-165.

Goldman-Rakic, P. S. (1991). Prefrontal cortical dysfunction in schizophrenia: The relevance of working memory. In B. J. Carroll \& J. E. Barrett (Eds.), Psychopathology and the brain (pp. 1-23). New York, NY: Raven Press.

Gooding, D. C., \& Tallent, K. A. (2002). Spatial working memory performance in patients with schizoaffective psychosis versus schizophrenia: A tale of two disorders? Schizophrenia Research, 53, 209-218. doi: 10.1016/S0920-9964(01)00258-4 
Harvey, P. D., Koren, D., Reichenberg, A., \& Bowie, C. R. (2006). Negative symptoms and cognitive deficits: What is the nature of their relationship? Schizophrenia Bulletin, 32, 250-258. doi:10.1093/schbul/ $\operatorname{sbj} 011$

Hashimoto, R., Lee, K., Preus, A., McCarley, R. W., \& Wible, C. G. (2010). An fMRI study of functional abnormalities in the verbal working memory system and the relationship to clinical symptoms in chronic schizophrenia. Cerebral Cortex, 20, 46-60. doi:10.1093/cercor/bhp079

Javitt, D. C., Strous, R. D., Grochowski, S., Ritter, W., \& Cowan, N. (1997). Impaired precision, but normal retention, of auditory sensory ("echoic") memory information in schizophrenia. Journal of Abnormal Psychology, 106, 315-324. doi:10.1037/0021-843X.106.2.315

Kay, S. R., Opler, L. A., \& Fishbein, A. (1999). Positive and Negative Syndrome Scale (PANSS) rating manual. Toronto, Ontario, Canada: MultiHealth Systems.

Kayser, J., Tenke, C. E., Gates, N. A., Kroppmann, C. J., Gil, R. B., \& Bruder, G. E. (2006). ERP/CSD indices of impaired verbal working memory subprocesses in schizophrenia. Psychophysiology, 43, 237-252. doi:10.1111/j.1469-8986.2006.00398.x

Kayser, J., Tenke, C. E., Kroppmann, C. J., Fekri, S., Alschuler, D. M., Gates, N. A., . . Bruder, G. E. (2010). Current source density (CSD) old/new effects during recognition memory for words and faces in schizophrenia and in healthy adults. International Journal of Psychophysiology, 75, 194-210. doi:10.1016/j.ijpsycho.2009.12.001

Keselman, H. J. (1998). Testing treatment effects in repeated measures designs: An update for psychophysiological researchers. Psychophysiology, 35, 470-478. doi:10.1017/S0048577298000237

Menon, V., Anagnoson, R. T., Mathalon, D. H., Glover, G. H., \& Pfefferbaum, A. (2001). Functional neuroanatomy of auditory working memory in schizophrenia: Relation to positive and negative symptoms. NeuroImage, 13, 433-446. doi:10.1006/nimg.2000.0699

Nurnberger, J. I., Blehar, M. C., Kaufmann, C. A., York-Cooler, C., Simpson, S. G., Harkavy-Friedman, J., . . Reich, T. (1994). Diagnostic Interview for Genetic Studies: Rationale, unique features, and training. Archives of General Psychiatry, 51, 849-859.

Oldfield, R. C. (1971). The assessment and analysis of handedness: The Edinburgh Inventory. Neuropsychologia, 9, 97-113. doi:10.1016/00283932(71)90067-4

Overall, J. E. (1974). The Brief Psychiatric Rating Scale in psychopharmacology research. Modern Problems of Pharmacopsychiatry, 7, 67-78.

Park, S., \& Holzman, P. S. (1992). Schizophrenics show spatial working memory deficits. Archives of General Psychiatry, 49, 975-982.

Park, S., Puschel, J., Sauter, B. H., Rentsch, M., \& Hell, D. (1999). Spatial working memory deficits and clinical symptoms in schizophrenia: A 4-month follow-up study. Biological Psychiatry, 46, 392-400. doi: 10.1016/S0006-3223(98)00370-9

Perlstein, W. M., Carter, C. S., Noll, D. C., \& Cohen, J. D. (2001). Relation of prefrontal cortex dysfunction to working memory and symptoms in schizophrenia. American Journal of Psychiatry, 158, 1105-1113. doi: 10.1176/appi.ajp.158.7.1105

Perry, W., Heaton, R. K., Potterat, E., Roebuck, T., Minassian, A., \& Braff, D. L. (2001). Working memory in schizophrenia: Transient "online" storage versus executive functioning. Schizophrenia Bulletin, 27, 157176.

Smith, E. E., \& Jonides, J. (1999, March 12). Storage and executive processes in the frontal lobes. Science, 283, 1657-1661. doi:10.1126/ science.283.5408.1657

Spitzer, R. L., Williams, J. B. W., Gibbon, M., \& First, M. B. (1990). Structured Clinical Interview for DSM-III-R: Patient edition. Washington, DC: American Psychiatric Press.

Stevens, A. A., Donegan, N. H., Anderson, M., Goldman-Rakic, P. S., \& Wexler, B. E. (2000). Verbal processing deficits in schizophrenia. Journal of Abnormal Psychology, 109, 461-471. doi:10.1037/0021843X.109.3.461

Stevens, A. A., Goldman-Rakic, P. S., Gore, J. C., Fulbright, R. K., \& Wexler, B. E. (1998). Cortical dysfunction in schizophrenia during auditory word and tone working memory demonstrated by functional magnetic resonance imaging. Archives of General Psychiatry, 55, 10971103. doi:10.1001/archpsyc.55.12.1097

Tulsky, D., Zhu, J., \& Ledbetter, M. F. (1997). WAIS-III-WMS-III technical manual. San Antonio, TX: Psychological Corporation.

Wechsler, D. (1981). The Wechsler Adult Intelligence Scale-Revised manual. San Antonio, TX: Psychological Corporation.

Wechsler, D. (1987). Wechsler Memory Scale: Revised manual. New York, NY: Psychological Corporation.

Wechsler, D. (1997). Wechsler Adult Intelligence Scale-Third Edition (WAIS-III). San Antonio, TX: Psychological Corporation.

Wexler, B. E., Donegan, N., Stevens, A. A., \& Jacob, S. A. (2002). Deficits in language-mediated mental operations in patients with schizophrenia Schizophrenia Research, 53, 171-179. doi:10.1016/S0920-9964(01) 00194-3

Wexler, B. E., Stevens, A. A., Bowers, A. A., Sernyak, M. J., \& GoldmanRakic, P. S. (1998). Word and tone working memory deficits in schizophrenia. Archives of General Psychiatry, 55, 1093-1096. doi:10.1001/ archpsyc.55.12.1093

Wible, C. G., Lee, K., Molina, I., Hashimoto, R., Preus, A. P., Roach, B. J., . . Lauriello, J. (2009). fMRI activity correlated with auditory hallucinations during performance of a working memory task: Data from the FBIRN consortium study. Schizophrenia Bulletin, 35, 47-57.

Wynn, J. K., Sugar, C., Horan, W. P., Kern, R., \& Green, M. F. (2010). Mismatch negativity, social cognition, and functioning in schizophrenia patients. Biological Psychiatry, 67, 940-947.

Received January 20, 2010

Revision received August 6, 2010

Accepted August 17, 2010 